\title{
CIRCULACIÓN ENTRE ALTA FUNCIÓN PÚBLICA Y EMPRESARIADO: EL CASO ARGENTINO EN PARTICULAR
}

\author{
Nicolás Vidal \\ Universidad de Buenos Aires
}

http://dx.doi.org/10.5209/rev_NOMA.2014.v43.n3.49284

\begin{abstract}
Resumen.- Este artículo propone valorar el concepto paretiamo de circulación para explorar la relación que se da entre el estado y la burguesía en las sociedades modernas analizando, como ejemplo, el paso desde la alta función pública a la esfera privada de hombres que participan en organizaciones patronales como Unión Industrial Argentina (UIA). Se interesa además en observar algunas particularidades de las elites en la Argentina y analizar dos casos prosopográficos que dan cuenta de la circulación mediante diferentes formas como son los desplazamientos y las conversiones. Al estudiar las historias de vida encontraremos dos tipos de representantes en la UIA, por un lado los propietarios de las empresas y, por otro, los ejecutivos de las mismas.
\end{abstract}

Palabras clave.- Circulación, elites, UIA, propietarios, gerentes.

Abstract.- This article proposes to appreciate the paretian conception of circulation for exploring the relationship given between the State and the bourgeoisie in the modern societies; analyzing, for example, the step existent from the upper-level public service to the private sphere of men who participate in trade associations as the Argentine Industrial Union (UIA). It is also interested in observing some particularities from the elites in Argentina, and analyzing two prosopographical cases that account for circulation through different ways, as they are displacements and conversions. Studying the history of lives, we will find two kinds of representatives at the UIA; on one side, the owners of the enterprises, and on the other hand, their executives.

Keywords.- Circulation, elites, UIA, owners, managers.

\section{Introducción ${ }^{1}$}

La verdadera conversión, es un movimiento profundo, por el cual el alma entera se gira en una dirección totalmente nueva, cambia de posición, de asiento y modifica, entonces, su punto de vista acerca del mundo (Durkheim, 1990:37)

En el marco de la sociedad capitalista moderna y sus distintas formas de organización gubernamental surge la pregunta sobre la igualdad que tienen las diferentes clases a la hora de imponer sus reivindicaciones a la sociedad en general. ¿Cuál es la

\footnotetext{
${ }^{1}$ Este articulo se desarrolla en el marco del proyecto Ubacyt número: 2002009030082 "Sociabilidades patronales y ética económica en la argentina contemporánea, la UIA" bajo la dirección de Luis Donatello, radicado en la Carrera de Sociología de la Facultad de Ciencias Sociales de la Universidad de Buenos Aires.
} 
influencia que las distintas clases pueden tener en la orientación económica y política de los gobiernos? ¿Son las sociedades democráticas del siglo XXI gobernadas por y para todos o hay una clase que impone su visión particular del mundo transformándola en general a través del control del estado?

En un plano individual quiénes son los que gobiernan y cuáles son sus motivaciones y experiencias que los transforman en conductores tanto en el estado como en la industria. La cita de Emile Durkheim, destacada más arriba, nos lleva a pensar en los cambios que desarrollamos los individuos en nuestras vidas y plantea a la conversión como algo radical, totalmente nuevo. ¿Es posible que los hombres y mujeres tengan "verdaderas conversiones"?, o ¿solo podemos desplazarnos de un lugar a otro pero siempre conservando nuestras estructuras subjetivas?

En este trabajo nos interesará abordar la relación que se da entre los grandes empresarios y la política. Cómo se pasa por una puerta giratoria, en términos de Michael Offerlé (2009), entre la alta función pública y la esfera privada y como eso puede significar ventajas a las empresas desde donde proviene el funcionario. Para comprender estos saltos parece conveniente no solo ver cómo se comportan los individuos que pasan la muralla entre lo público y lo privado sino también detenerse en la relación que existe entre el estado y las grandes empresas, o para ser más especifico, entre el estado y la burguesía en el sistema capitalista y, por último, al ser esta burguesía heterogénea como se desenvuelve particularmente en la Argentina.

El objetivo principal de este trabajo es plantear el vínculo que existe entre el mundo empresarial y la política.

Tanto empresarios como altos cargos gerenciales en la industria mantienen contacto con el estado. Este contacto es través de las organizaciones patronales como la UIA o formando parte del gobierno, ocupando puestos en el estado, negociando directamente a través de su empresa subsidios, facilidades impositivas, etc.

Desde una mirada circunscripta a las personas como sujetos sociales podemos hablar de circulación que se da en el paso de una posición a otra, del ámbito privado al público o a la inversa.

Intentaremos describir distintos mecanismos de circulación, específicamente los de conversión, cuando los sujetos cambian (formas) y creencias en su actuar, o como desplazamiento, cuando, solamente, se da una reclasificación profesional.

Existen tres núcleos relevantes en la bibliografía a tratar y son: la relación entre estado y capital y el caso argentino en particular; los desplazamientos o passe muraille (atravesar muros); la heterogeneidad patronal. 
Espacialmente este artículo se organizará planteando un estado de la cuestión que propondrá un apartado para cada núcleo relevante que describimos anteriormente; luego en una segunda sección se propondrá una breve exposición de las entrevistas que se realizaron.

\section{Estado de la cuestión. La relación entre Estado y capital: el caso argentino en particular.}

Pensar en el estado como un imparcial en el ámbito de la lucha de clases es irreal en términos marxistas. Dice Lenin en uno de sus escritos más trascendentes que para Marx, el Estado es un órgano de dominación de clase, un órgano de opresión de una clase por otra, es la creación del "orden" que legaliza y afianza esta opresión, amortiguando los choques entre las clases" (2004:23). Si tomamos esta definición entendemos que el Estado es representante del capital y la burguesía ocupa con sus hombres, sean burgueses o gerentes, lugares clave en el aparato estatal.

Michel Offerlé (2009) en Sociología de las organizaciones patronales explica que la patronal no es un actor como otros y tiene la característica de poder imponer y manipular en la política y las políticas públicas. En estos términos es que nos preguntamos cómo se dan las relaciones, influencias, manipulaciones entre las grandes empresas capitalistas y los estados.

Aquí expondremos algunas ideas de Ana Castellani al respecto (2009a; 2009b). La autora busca las relaciones existentes entre la intervención estatal en la economía y el grado de participación del empresariado en tales decisiones para generar ámbitos privilegiados de acumulación. Distingue entre los conceptos de ámbitos de acumulación estratégicos y ámbitos privilegiados de acumulación (APA). El primero es característico de un país donde se intenta la implementación de un proyecto de desarrollo sostenido, como por ejemplo en Brasil en los últimos 50 años, donde tanto empresarios como estado toman políticas claras y a largo plazo. El segundo, es el característico de nuestro país y prioriza el otorgamiento de cuasi rentas de privilegio (Notcheff, 1994) a grupos empresarios que privilegian la ganancia sustantiva en el corto plazo, aprovechando las distintas "burbujas" que se generan, en detrimento de una estrategia a largo plazo donde se pueda obtener otro tipo de crecimiento.

Los APA son "espacios donde las empresas privadas involucradas obtienen ganancias extraordinarias derivadas de la existencia de privilegios institucionalizados y no institucionalizados generados por el accionar estatal" (2009a: 23). La "elite" económica intenta sostener ámbitos privilegiados de acumulación en el mercado y, para eso, necesita 
de ayuda estatal o ayudarse desde el estado ocupando obtener puestos clave en el gobierno para poder acaparar beneficios o privilegios.

Podríamos incluir a modo de ejemplo el funcionar de la presión que ejerce la burguesía sobre las políticas estatales. Mark Smith (2007) en su artículo, "Le trois cas de figure de pression", muestra la desigualdad en el acceso al congreso estadounidense. Los temas a tratar, los informes e inscripciones en el parlamento norteamericano impuestos por los grupos empresarios equivalen al $45 \%$ de los mismos. Este porcentaje es el nivel de lobbying que detentan los grandes grupos económicos (hay que recordar que esta actividad es legal y está reglamentada en EE.UU). En esta información se muestra como a fuerza de dinero, las empresas, pueden imponer sus temas sobre los de los demás grupos y asociaciones como sindicatos $u$ ongs.

Jorge Schvarzer (1996) analiza el caso particular de la UIA en su artículo "La elite empresaria en la Argentina: la Unión Industrial Argentina". Extraeremos algunas ideas de aquí que nos ayuden a comprender esta entidad de la que forman o formaron parte los entrevistados a los que nos referiremos más adelante.

Para el autor, la UIA nace prematuramente en 1887 constituida por un reducido grupo de industriales que formaban parte de la clase dominante local. Este grupo es de carácter elitista y la Unión toma el carácter de un club exclusivo más que de una asociación corporativa. La UIA es controlada, históricamente, por grandes empresarios que imponen sus intereses particulares y obstruyen el ascenso de pequeños y medianos empresarios que quieran llegar a dirigirla. Estos intereses particulares, explica Schvarzer, son, generalmente, concordantes con el resto de la clase dominante. Dice el autor que "La conclusión que surge del análisis de sus posiciones es que la UIA no llego nunca a diferenciarse estratégicamente de las perspectivas sostenidas por la clase dominante local, de la cual formaba parte" y que "hubo diferencias que no pueden ignorarse pero que se referían mas a aspectos circunstanciales y de coyuntura que a temas de fondo". (Schvarzer, 1996:127)

Esta elite empresaria que maneja la UIA se asocia con sectores conservadores y dominantes, sin representar una alternativa industrialista para el desarrollo del país sino más bien como un medio para defender sus propios intereses y no los de los industriales en general. Explica Schvarzer que es una "dirigencia de carácter elitista, basada en el poder económico y las relaciones político sociales de sus conductores que en el objetivo de representar al sector" (Schvarzer, 1996:125)

\section{Heterogeneidad patronal}

Antes de continuar nos gustaría introducir algunas ideas de Vilfredo Pareto sobre la teoría de las elites. Para este autor las elites están formadas por hombres que se destacan, 
que tienen cualidades excepcionales en su ámbitos particulares como pueden ser la cultura, la economía, la política, etc. Les atribuye un valor cualitativo. El poseer estas cualidades les confiere poder y prestigio en su actividad.

Dice Pareto, en la introducción de su libro Les systemes socialistes, que si dispusiéramos a los hombres según su grado de influencia y de poder político y social en la mayoría de las sociedades los que ocupan los sitios más importantes en ellos coinciden con los figuran en la en la cima de la distribución de la riqueza. Dice el autor que '”las clases declaradas superiores son también, generalmente, las más ricas“. (Pareto, 1902:8)

Agrega además que "estas clases representan una elite, una aristocracia (relacionado etimológicamente como "mejor"). Tanto que si el equilibrio social es estable, la mayoría de los individuos que la componen aparecerán eminentemente dotados de ciertas cualidades, buenas o malas que, por cierto, garantizaran el poder". (Pareto, 1902:8) Las aristocracias no duran. Ellas son golpeadas por la decadencia tarde o temprano. (Pareto, 1902:9)

Destaca en su libro Forma y equilibrio sociales que estas elites y las clases gobernantes no son homogéneas, no tienen una unidad concreta ni tienen una única voluntad. (Pareto, 1980:162)

Desde una mirada marxista y volviendo sobre nuestros pasos, la cita de Lenin más arriba formulada podría especificarse con algunas ideas de Nicos Poulantzas (1969) en su libro, Poder político y clases sociales en el estado capitalista, donde nos explica que al interior del bloque de poder, que detenta el estado, existen divergencias y distintas posturas entre las clases o fracciones de clase que lo conforman. Para el autor la burguesía no constituye una clase sujeto sino que el bloque de poder es una unidad contradictoria de las clases o fracciones de clase dominantes. (1969: 388,391) La clase o fracción que prevalece sobre las demás, explica Poulantzas, capta los intereses de las otras que forman parte del bloque de poder para mantener la hegemonía, con ayuda del estado que, pese a ser factor de unidad del mismo, se corresponde estrictamente con los intereses específicos de la primera.

Para Jean Garrigues (2007) la homogeneidad patronal no existe porque siempre se encuentran intereses contrapuestos en las diferentes ramas capitalistas, esto, a su vez, genera que no haya unidad en las centrales empresarias. Continuando con lo expuesto por Jean Garrigues, Castellani (2009:36) explica que la "elite" no es un sector social homogéneo. Está integrado por diferentes fracciones que, en más de una ocasión, tienen intereses y conductas contrapuestos. 
Centrándonos en el caso argentino puede observar esta heterogeneidad en los diferentes nucleamientos patronales y sus distintos objetivos y puntos de vista. No es lo mismo la política llevada adelante por la UIA (Unión Industrial Argentina) que por la SRA (Sociedad Rural Argentina) u otras entidades como son Federación Agraria o la CGE (Confederación General Económica) de antaño. Quien logre imponerse como grupo hegemónico intentara convertir en generales su visión particular del mundo.

Al observar el nucleamiento patronal que es eje de nuestro trabajo, la UIA, podemos integrar algunas ideas de María Virginia Dossi (2012) que hace un extenso y muy buen trabajo sobre la historia de Unión Industrial Argentina y las diferentes formas de representación de las que disponen los empresarios para expresar sus demandas. Nos sirve su trabajo para entender que la heterogeneidad de la burguesía no se da, sólo en las disputas por el poder estatal, sino también al interior de las organizaciones patronales como la UIA. En ella confluyen distintos lineamientos internos, algunos sectores de carácter industrialista y mercado internista con otros alineados con los sectores agroindustriales que privilegian una economía exportadora de materias primas. Nos dice Dossi que: " Los empresarios constituyen un conjunto complejo y heterogéneo y es necesario comprenderlo para dar cuenta de la realidad de este sector y de los procesos de formación de representación de intereses y de acción corporativa empresaria que se producen en su interior". (2012: 20)

\section{Circulación: Desplazamientos y conversiones}

Para Vilfredo Pareto el grupo dominante no es fijo, está en constante movimiento y requiere nuevos elementos para engrosar sus filas. A este fluir de personas desde las clases que no gobiernan, inferiores, hacia el gobierno, el autor, lo denomina circulación.

La circulación se da para el sociólogo italiano por las "bajas en las guerras, por la menor cantidad de nacimientos en las clases superiores y la degeneración de algunos elementos que la componen. No puede subsistir sin la eliminación de estos elementos y el aporte de otros nuevos". (Pareto, 1902: 8) Este mecanismo es importante porque "un simple retraso en la circulación puede tener el efecto de aumentar el número de elementos que se degeneran dentro de las clases poseedoras y de aumentar, del otro lado, la cantidad de elementos de merito en las clases inferiores" (Pareto, 1902:11)

En resumen, las clases gobernantes o elites requieren de nuevos elementos que provienen de las más preparadas de las clases inferiores para afianzar su predominio, al mismo tiempo, evitan que estas disputen su hegemonía desde una posición de no gobierno. Este ascenso social que aquí se presenta es una de los puntos a destacar a la hora de analizar las trayectorias individuales de los entrevistados. 
Michel Offerlé en su artículo "chef d'entreprise, patronat et politique" explora la relación entre los jefes de empresa y la política. Como algunos entran a la arena política por una "puerta giratoria" que los conecta entre el congreso y los sillones de sus compañías. (Offerlé, 2007)

Poniendo como ejemplo a Francia dice que más allá que la política se está convirtiendo en una actividad autónoma, desarrollada por profesionales liberales, los jefes de empresa están sobrerrepresentados en el congreso y los ministerios en comparación a su incidencia en la población activa.

Si nos recostamos en la trayectoria individual de los funcionarios/empresarios podemos preguntarnos el porqué del cambio entre lo público y lo privado, por ejemplo. En el libro "Reconversiones militantes" se intenta desentrañar como en distintas esferas sociales se producen desplazamientos, transformaciones y conversiones de hombres y mujeres que atraviesan murallas (passe muraille) (Brunet, 2005) para saltar entre distintas esferas sociales o cambiar de posición dentro de una misma actividad.

Se analizan casos como, por ejemplo, de militantes franceses de mayo del '68 y sus distintas trayectorias donde algunos pasaron de ser trotskistas, bregando por la revolución socialista, a "resignarse" a obtener un buen puesto en la función pública o en la dirección de empresas; lo mismo para algunos sindicalistas reconvertidos por sus propios patrones en colegas; o, el caso, como el que describe Philippe Brunet (2005), de una militante ecologista reconocida por su actuación en Francia durante la crisis de la energía nuclear provocada luego del accidente de Chernóbil que se transforma en funcionaria de medioambiente .

Las tres perspectivas para analizar las reconversiones, según Silvie Tyssot son: Desplazamientos; Transformaciones y valoraciones de recursos específicos; recomposiciones identitarias (conversión)

1. Ciertos contextos específicos favorecen la reclasificación profesional. Reconvertirse, para militantes, no es solamente, ni necesariamente, cambiar las ideas, es también desplazarse en el espacio social.

2. Estas trayectorias, de los militantes de mayo del sesenta y ocho, solo son posibles por la conversión de un capital militante o político que supone una transformación conforme a las reglas de los nuevos espacios donde están invertidas. Del capital asociativo la capital colectivo.

3. La movilización del saber-hacer (savoir faire) pasa a menudo por un trabajo discursivo que evacue toda conflictividad en provecho de una visión más pacifica del mundo social. 
4. Hay lugares, como la enseñanza para los militares, donde se pueden aprovechar capacidades especificas como la oratoria para insertarse en "otro" espacio. Estos son "restablecimientos simbólicos" para Bourdieu.

5. El termino de conversión, en su sentido religioso, indica un tercer elemento de los objetos aquí estudiados. Las reconversiones implican desplazamientos en el espacio social pero aparecen también como "conversiones", es decir transformaciones que afectan también las maneras de ser y de pensar.

\section{Desarrollo del caso ${ }^{2}$}

Para escribir este artículo se realizaron y se consultaron entrevistas a hombres que forman o formaron parte de la Unión Industrial Argentina (UIA) tanto de su comité ejecutivo como de su junta directiva.

La entrevista de referencia se realizó a un directivo de una empresa automotriz que ha tenido un cargo en la UIA y en ADEFA (Asociación de Fabricas de Automotores) y cargos en la gestión pública a lo largo de su carrera profesional.

El entrevistado, al que llamaremos Marcelo, es un hombre de mediana edad, de no más de cincuenta años, con estudios universitarios y un máster en finanzas en su haber. Oriundo del interior del país, con padres profesionales y socializados en el mundo católico decidió estudiar en Buenos Aires una carrera que a la larga lo terminaría vinculando con la industria automotriz.

Durante un largo período de su carrera profesional se desempeño en el ámbito estatal con puestos relacionados con la industria y el comercio internacional. En el ámbito privado tenemos información de su paso por automotrices que se produjeron antes y después de pasar a la función pública.

Marcelo proviene de una familia donde la política ha sido parte de sus vidas y el mismo es afiliado al partido justicialista.

Otros entrevistados, Simón y Gerardo son de otra generación, los dos son mayores de 70 años. El primero se encuentra retirado de la actividad empresarial (aunque sigue trabajando por hobbie en otra) y el segundo todavía está al mando de la cámara que nuclea su actividad.

\footnotetext{
${ }^{2}$ En el proyecto de referencia, estamos desarrollando entrevistas cualitativas a los fines de reconstruir las biografías de los miembros de la junta directiva y el comité ejecutivo de la UIA entre 2001 y la actualidad. Dadas las características de dicha metodología, no podemos inferir resultados válidos estadísticamente, sino comprender diferentes modalidades de articulación entre las dimensiones que emergen como significativas del trabajo empírico. En ese sentido, es que los casos que tomamos exponen lógicas relevantes del fenómeno que nos ocupa.
} 
En el caso particular de Simón nació en la Ciudad de Buenos Aires y vivió toda su vida allí. Su padre era profesional independiente e inculco en él una formación laica pues su familia era judía pero no religiosa. Estudio en colegios y universidades del estado y su carrera profesional la realizo en pocas empresas hasta que tuvo la oportunidad de formar parte del capital accionario de una que, a la larga, seria en su totalidad suya y en la que invirtió gran parte de su vida laboral.

No tuvo cargos en el estado nacional, como así tampoco tuvo actividad partidaria en su vida académica y profesional, pero, si participó activamente de la Unión Industrial Argentina con diversos cargos en su órgano administrativo y fue presidente de la cámara que nuclea a las empresas de su misma rama industrial.

Estas entrevistas nos son de utilidad para comparar algunos aspectos con Marcelo, sobre todo en lo respectivo a los tiempos en que se desarrollaron sus carreras vinculadas con distintas etapas históricas en el país.

Podemos decir tanto de Marcelo como de Simón que, en términos paretianos, son elementos que la elite ha reclutado entre las clases no gobernantes o inferiores para cubrir lugares que no pueden completar ellos mismos. Los dos provenientes de familias de sectores medios que lograron un ascenso social mediante la circulación entre diferentes espacios. En el caso del primero mediante un desplazamiento como gerente o tecnócrata que es convocado tanto para el sector privado como el publico para representar los intereses de la elite y, el segundo, convertido ya a miembro pleno de la elite y capitalista, dueño de una empresa reconocida en su sector, que formó parte muchos años de la UIA para defender sus intereses y los de sus pares a través de esta organización corporativa que es además uno de los medios mediante los cuales negocian-presionan al estado.

En síntesis nuestro entrevistado de referencia hay desplazamiento según las oportunidades que va generando/recibiendo entre lo privado y lo público y en el otro caso analizado hay conversión, se pasa de gerente a dueño. Cabe mencionar que Simón fue gerente en un pequeño número de empresas antes de convertirse en dueño.

Si tomamos lo expuesto por Miliband en El estado en la sociedad capitalista esta circulación de las elites se da generalmente con gerentes provenientes de las clases profesionales, como en el caso de nuestros entrevistados, y propietarios y, no muy frecuentemente para no decir casi nunca, de las clases trabajadoras. (Miliband,)

Como comentamos más arriba, Marcelo está afiliado a uno de los partidos históricos de nuestro país como es el Partido Justicialista continuando una vinculación con la política familiar. Su padre estuvo muy ligado a ella en la ciudad de donde son originarios. 
Sus puestos en el Estado los ocupo en gobiernos peronistas y entendemos que hay aquí una relación que excede este trabajo pero sería interesante seguir investigando como posible motivación en la circulación entre estado y ámbito privado de industriales argentinos.

En otras de las entrevistas se hacía referencia al actual presidente de la UIA, Ignacio De Mendiguren, como ejemplo de positivo para la entidad porque llego a ser Ministro de Industria en el gobierno de Eduardo Duhalde y que la entidad históricamente se personifico mas como oyente que como actor en la vida económica argentina a diferencia de la Sociedad Rural.

Aquí vemos como se encuentra una visión positiva de parte de los empresarios que haya otros que ocupen lugares en el gobierno e instalen sus demandas particulares. Se pide una mayor participación y representación como si lo hace la SRA históricamente y debería ser un modelo a imitar a través de hombres como De Mendiguren, Paolo Rocca u otros.

Nos parece importante pensar en esta diferencia que se marca entre gerente y dueño en los entrevistados. Marcelo es un gerente o elemento de una tecnocracia que se encarga de dirigir los destinos de una empresa multinacional o una secretaria en el estado; en cambio Simón ha pasado de gerente a dueño, ha completado la circulación desde hijo de profesionales a miembro pleno de la elite. ¿Existe una diferencia entre los intereses que puedan representar uno y otro?

Mariana Heredia (2010) incluye en uno de sus artículos, donde analiza las vicisitudes que se presentan al intentar realizar una distinción de las llamadas elites explorando distintas formas de caracterizarlas, un esquema desarrollado por Robert Erikson y John Goldthorpe que, dentro de las denominadas teorías de la estratificación, trabajan sobre ello. Este esquema propone determinar a las elites por distintos recursos, entre ellos la educación, y no por la propiedad o no de los medios de producción. El esquema ubica a "capitalistas" y "altos ejecutivos" formando parte de las elites o clases altas. Los primeros se dividen a su vez en propietarios y socios gerentes y, los segundos, son los ejecutivos que tienen por objeto administrar empresas privadas e instituciones públicas.

Ralph Miliband en su libro El estado en la sociedad capitalista al hablar de las elites y la clase dominante intenta comprender el fenómeno de los gerentes que aparecen como manifestación de la separación entre la propiedad y el control de las empresas en el sistema capitalista. Retomando esta pensamiento marxiano llega a la conclusión que, más allá de la diferencia, dueños y gerentes sobreponen sus diferencias por su fundamental comunidad de intereses. Dice el autor que "los factores que asemejan a los patronos de estilo familiar y a los gerentes profesionales son muchos más fuertes que los elementos que los distinguen: así los primeros, como los segundos, son dirigentes capitalistas". (Miliband, 1988:39) 
Una de las diferencias que encuentro con otros entrevistados es en la edad, mientras que Marcelo es directivo en una empresa importante de su sector y se encuentra en plena actividad, los otros entrevistados están al final de su carrera profesional o ya son retirados y, en general, son dueños de empresas.

Las automotrices en la Argentina, como sabemos, son filiales de empresas multinacionales en su totalidad y aun en puestos jerárquicos y de toma de decisiones se sigue siendo un trabajador de un consorcio capitalista extranjero.

Otros entrevistados son o fueron presidentes de empresas de capital argentino de las que son propietarios de la mayoría accionaria.

Otra diferencia importante entre Juan y los demás entrevistados son los lugares de residencia a lo largo de su vida.

Marcelo, de 50 años aproximadamente, ha vivido en distintos lugares de nuestro país y del exterior, en total cinco ciudades diferentes.

Arribó a Buenos Aires desde el interior del país para seguir su carrera universitaria y luego su carrera y su trabajo lo han llevado a residir en EE.UU y Europa Luego, nuevamente el trabajo, lo trajo a Buenos Aires donde vive y formó su familia.

Richard Sennet en su libro la corrosión del carácter hace un análisis interesante de cómo cambia el mundo laboral, familiar y académico de una generación a otra poniendo como ejemplo a una familia "típica" estadounidense con un padre que trabajo desde la década del sesenta y su hijo que se inserto en el mundo laboral en los noventa.

Resulta interesante ver cómo cambia el mundo de la organización fordista de la producción a la presente mucho mas flexibilizada y eso repercute en la manera como organizamos nuestras vidas.

Marcelo para desarrollar su carrera debió viajar y radicarse en distintos lugares del mundo "aprovechando oportunidades" y adaptándose constantemente los nuevos tiempos, mientras que los otros entrevistados formaron sus carreras en una o pocas empresas y terminaron dirigiendo la misma o adquiriendo una propia.

La carrera laboral de Simón y su paso a dueño se dio en las décadas de los sesenta y setenta mientras que la de Marcelo se desarrollo a partir de los noventa.

Entendemos que este punto es clave en las diferencias en las trayectorias de los entrevistados porque los momentos históricos particulares en que desarrollaron sus carreras son diferentes y las ramas de actividad también al ser el sector automotriz fuertemente acaparado por empresas de capital extranjero a diferencia del sector de la empresa de Simón donde existe una parte de las empresas de capital nacional aun hoy en día. 
Durante el gobierno de Arturo Frondizi (1958-1962 fijarse) se extiende la liberación a la entrada de capitales extranjeros que genera que un gran número de empresas multinacionales se radiquen en el país y, entre ellas, algunas automotrices como Renault y Fiat que comienzan a producir

La industria automotriz es de las más dinámicas de la Argentina en la actualidad y es una de las pocas ramas industriales que se encuentran entre las principales exportadoras junto a los tubos de acero sin costura. Según un dato de 2008 solo Ford y Siderca logran mezclarse entre las primeras veinte exportadoras del país que están dominadas por el complejo sojero e extractivas como la petrolera. (Teubal, 2011)

En la década del noventa con el boom del endeudamiento tanto del estado como de los particulares la industria automotriz se vio favorecida como destinataria de un régimen especial de promoción y protección. El "régimen automotriz" fue constituido entre 1990 y 1992 con una serie de decretos que exceptuaron a dicha industria de la liberación del mercado y desregulación de la economía de los años noventa. Esta creció exponencialmente entre 19990 y 1995 llegando al record de patentamientos en 1994 con más de 500 mil autos registrados. Para Sebastián Etchemendy ADEFA y los sindicatos del sector como SMATA tuvieron un gran poder de lobby durante la formulación y emisión de los decretos que regularon al sector. (Etchemendy, 2001)

En este contexto es que nuestro entrevistado, Marcelo, fue parte del gobierno y vemos entonces la relación importante que se da entre la industria automotriz y el gobierno de turno y el poder de lobby e injerencia en el estado que logran los grupos económicos que dominaban la industria en ese momento, donde todavía existían algunos de capital nacional como SEVEL, de Franco Macri, que se irían extranjerizando nuevamente en esa década.

Por último nos gustaría resaltar las distintas estrategias personales de los entrevistados respecto a su participación en la institución que los representa, la Unión Industrial Argentina. Marcelo nos comentaba que su carrera fue guiada por las oportunidades que se presentaron según la coyuntura económica de país y al hecho de formar parte de la UIA lo entiende como una necesidad laboral. A diferencia de otros entrevistados, como Simón y Gerardo, en los que se nota una convicción en pertenecer a órganos de representación empresarial como retribución a su buen desempeño dentro de las federaciones o cámaras de la industria que representan a sus empresas.

\section{Conclusiones}

Intentaremos exponer lo que, a nuestro entender, son algunas consecuencias políticas y económicas resultantes de la relación entre empresariado y política que describimos en este trabajo. 
Nosotros nos hemos referido a dos tipos de vinculaciones entre las empresas y la política que son: la circulación entre los ámbitos públicos y privados de cierta tecnocracia formada por empresarios gerentes, y la relación a través de órganos de representación sectorial como es el caso de la UIA.

Creemos innegable la existencia de estos vínculos estrechos donde, en el caso de la circulación de tecnócratas, hay un perfil de empresario que va y viene entre la gestión pública y la privada, y que, estando en el estado, trabaja no solo para la nación sino también generando o manteniendo buenas relaciones con las empresas que luego pueden convocarlos al salir de la función pública.

En el caso de los órganos de representación estos intentaran defender sus intereses ante el estado tratando de imponer su modelo de país. Pensando en tres grandes sectores económicos como son la industria, el sector financiero y parte del heterogéneo mundo del campo y sus órganos de representación: UIA, SRA (Sociedad Rural Argentina), ADEBA (Asociación de Bancos Argentinos) podemos decir que tienen distintos grados de representación política en el gobierno. Las disputas por imponer los intereses particulares de estos grupos son donde se define la matriz económica del país históricamente o por lo menos de 1930 en adelante. Para Schvarzer (1996), como hemos visto, la UIA no constituye una alternativa a la clase dominante local ya que forma parte de ella y, aun con diferencias circunstanciales, concuerda en general con el modelo de país. Ralph Miliband va aún más lejos asegurando que las clases dominantes tienen conciencia de clase, en términos marxistas, porque "más allá de todas sus diferencias y desacuerdos, los ricos y los propietarios han estado siempre fundamentalmente unidos, en defensa del orden social que les otorgaba sus privilegios" (Miliband, 1988:48).

Estos tipos de vinculaciones, a nuestro entender, determinan muchas veces la "agenda" del estado y la conformación de lo que Castellani (2009) denomina Ámbitos Privilegiados de Acumulación (APA).

Las consecuencias económicas de vínculo, a primera vista, parecen de gran beneficio para los capitalistas que logran, no solo la formación de APA, sino también la invisibilización de este proceso a la sociedad toda. No es común ver, escuchar, ni leer en los medios de comunicación acerca de los grandes negocios que realizan los empresarios a costa del estado, ni tampoco sobre las subvenciones multimillonarias que reciben.

Volviendo ejemplo de la industria automotriz, desde la salida de la convertibilidad hasta el presente esta ha mantenido su posición de privilegio dentro de la debilitada industria argentina post dictadura y década del noventa que la ha llevado a nuevos records 
de producción y patentamientos. En 2011, según datos de ADEFA ${ }^{3}$, la producción llegó a 830 mil vehículos.

La diferencia que podemos encontrar es que las terminales automotrices en el presente son todas filiales de sus casas matrices y no empresas argentinas, como era, por ejemplo, SEVEL de Franco Macri, que adquirían los derechos de representación en el país. A partir de los noventa, como vimos más arriba, la industria automotriz pasa a ser un sector atractivo para las inversiones y claves en el presente para estas empresas transnacionales en sus beneficios mundiales. Sea por el crecimiento de la región y/o por la contracción de los mercados centrales.

Esto es muy importante si pensamos en quienes dirigen estas filiales (empresarios gerente) y como, al ser empresas muy importantes a nivel mundial, pueden ejercer una presión diferente hacia el estado. La apertura al capital extranjero, que comenzó a finales de la década del cincuenta del siglo pasado y que se ha intensificado desde los años setenta, con gobiernos que priorizan políticas neoliberales de desregularización y apertura económica, ha alcanzado un punto en que la extranjerización de la economía argentina es tan fuerte que desaparecieron muchas pequeñas y medianas empresas y empresas líderes en distintos sectores industriales cambiaron a manos extranjeras, como, por ejemplo, Cervecería y Maltería Quilmes de la familia Bemberg o la cementera Loma Negra que pertenecía a Amalia Fortabat.

Esto, a nuestro entender, reduce la cantidad de empresarios dueños como Simón e incrementa la de empresarios gerentes como Marcelo. Excede a este trabajo comprender como pudo esto modificar la constitución de la burguesía nacional en los últimos 60 años.

\section{Referencias bibliográficas}

BRUNET, P. (2005), "La CRII-RAD: une laboratoire passe-muraille entre militantisme et professionnalisme", en Tyssot, Sylvie, Gaubert, Christophe, Lechien, Marie-Helene, Reconversions Militantes, Pulim, Limoges.

CASTEllani, A. (2009a), Estado, empresas y empresarios: la construcción de ámbitos privilegiados de acumulación entre 1966 y 1989, Buenos Aires, Prometeo libros.

- (2009b), "Estado y grandes empresarios en la Argentina de la post-convertibilidad", Cuestiones de Sociología (5/6), FAHCE-UNLP, ISSN 1668-1584.

\footnotetext{
${ }^{3}$ http://www.adefa.com.ar/v2/index.php, consultado el 4 de diciembre de 2012.
} 
Dossı, M. (2012), "La Unión Industrial Argentina: su organización y vinculaciones con el mundo de las corporaciones empresarias", Documentos de investigación social, número 19, Buenos Aires: IDAES-UMSAM.

DURKHEIM, É. (1990), L'évolution pédagogique en France, Paris, PUF.

ETCHEMENDY, S. (2001), "Construir Coaliciones Reformistas: La Política de las compensaciones en el camino argentino hacia la liberalización económica", Desarrollo Económico, Vol. 40, num. 16.

GarRigues, J. (2007), "Rivalités patronales et "influence" du patronat", Problemes Politiques et Sociaux, num. 937, 107.

HeREDIA, M. (2010), ¿La formación de quién? Reflexiones historiográficas, sociológicas y estadísticas sobre el modo de delimitar a las elites sociales en la Argentina: reunión internacional sobre formación de las elites, Buenos Aires, FLACSO,

LENIN, V. (2004), El Estado y la Revolución, Buenos Aires: Nuestra América.

MILIBAND, R. (1988), El estado en la sociedad capitalista, México, Editorial Siglo XXI.

NoCHTEFF, H. (1994), "Los senderos perdidos del desarrollo. Elite económica y restricciones al desarrollo en la Argentina”, en Azpiazu, Daniel y Nochteff, Hugo, El desarrollo ausente. Restricciones al desarrollo, neoconservadurismo y elite económica en la Argentina, Ensayos de Economía Política, Buenos Aires, Flacso.

OfFerté, M. (2007), "chef d'entreprise, patronat et politique", Problemes Politiques et Sociaux, N937, Paris, 98.

- (2009), Sociologie des organizations patronales, Paris: Editions La Decouverte.

Pareto, V. (1902), Les systemes socialistes, Paris, V. Giard \& E. Briere Libraires-editeurs.

- (1980), Forma y equilibrios Sociales, Madrid: Alianza Editorial.

Poulantzas, N. (1969), Poder político y clases sociales en el estado capitalista, México, Editorial Siglo XXI.

SCHVARZER, J. (1996), "Una elite empresaria en la Argentina: la Unión Industrial Argentina", Ensaios FEE, Fundación de Economía e Estatística S.E.H., año 17 num. 2, Porto Alegre.

SENNET, R. (2000), La corrosión del carácter, Barcelona, Editorial Anagrama.

SMIth, M. (2007), "Le trois cas de figure de pression", Problemes Politiques et Sociaux, N937, Paris, 113.

Teubal, M. y PALmisano, T. (2011), "El conflicto agrario: características y proyecciones". En Giarracca, N. y Teubat, M. (Coord), Del paro agrario a las elecciones de 2009. Tramas, reflexiones y debates, Buenos Aires, Editorial Antropofagia.

TYSSOT, S. (2005), "Les reconversions militantes", en Tyssot, Sylvie, Gaubert, Christophe, LECHIEN, M. (2009), Reconversions Militantes, Limoges, Pulim. 